TRANSACTIONS OF THE

AMERICAN MATHEMATICAL SOCIETY

Volume 352, Number 7 , Pages 3015-3027

S 0002-9947(00)02366-7

Article electronically published on February 24, 2000

\title{
A CRITERION FOR REDUCTION OF VARIABLES IN THE WILLMORE-CHEN VARIATIONAL PROBLEM AND ITS APPLICATIONS
}

\author{
MANUEL BARROS, ANGEL FERRÁNDEZ, PASCUAL LUCAS, AND \\ MIGUEL A. MEROÑO \\ Dedicated to the memory of Alfred Gray
}

\begin{abstract}
We exhibit a criterion for a reduction of variables for WillmoreChen submanifolds in conformal classes associated with generalized KaluzaKlein metrics on flat principal fibre bundles. Our method relates the variational problem of Willmore-Chen with an elasticity functional defined for closed curves in the base space. The main ideas involve the extrinsic conformal invariance of the Willmore-Chen functional, the large symmetry group of generalized Kaluza-Klein metrics and the principle of symmetric criticality. We also obtain interesting families of elasticae in both lens spaces and surfaces of revolution (Riemannian and Lorentzian). We give applications to the construction of explicit examples of isolated Willmore-Chen submanifolds, discrete families of Willmore-Chen submanifolds and foliations whose leaves are Willmore-Chen submanifolds.
\end{abstract}

\section{INTRODUCTION}

The Willmore-Chen variational problem is the natural extension to higher dimensions of the well known Willmore variational problem. It is associated with the Willmore-Chen functional

$$
\mathcal{W}(N)=\int_{N}\left(\alpha^{2}-\tau_{e}\right)^{\frac{n}{2}} d V
$$

defined on the space of compact $n$-dimensional submanifolds $N$ of a given Riemannian or pseudo-Riemannian manifold $P$. The terms appearing in the integrand of $\mathcal{W}$ are the mean curvature function $\alpha$ and the extrinsic scalar curvature $\tau_{e}$ of $N$ in $P$, which measures the difference between the scalar curvature of $N$ and a part of the scalar curvature of $P$ along $N$. The problem can be sketched as follows:

1. Find the critical points of $\mathcal{W}$, which we will call Willmore-Chen submanifolds.

2. Study the stability of critical points, which involves the determination of the minimal values of $\mathcal{W}$.

The importance of this problem partially comes from its invariance under conformal changes of the metric in the ambient space (see [9]). Thus $\mathcal{W}$ might also be called the

Received by the editors November 11, 1997 and, in revised form, June 25, 1998.

2000 Mathematics Subject Classification. Primary 53C40, 53A30, 58E30.

Key words and phrases. Willmore-Chen submanifold, Kaluza-Klein metric, elastic curves.

This research has been partially supported by DGICYT grant PB97-0784 and Fundación Séneca (C.A.R.M.) grant PB/5/FS/97. 
conformal total curvature functional. The classical Willmore functional corresponds with the case $n=2$. Any (compact) minimal surface in a standard sphere (in general, any zero mean curvature surface in a real pseudo-Riemannian space form) is automatically a Willmore surface. Examples of nontrivial Willmore surfaces in standard spheres are given in 3, 7, 10, 14 and 17. Examples of Willmore surfaces in nonstandard spheres can be found in [1] and [8], as well as in spaces with a pseudo-Riemannian global warped product structure in [2] and [4]. The first nontrivial examples of Willmore-Chen submanifolds in standard spheres were given in [6], and later in [4] for conformal structures associated with warped product metrics, and consequently on reducible spaces.

In this paper we deal with Willmore-Chen submanifolds in conformal classes associated with the so-called generalized Kaluza-Klein (Riemannian or pseudoRiemannian) metrics on principal fibre bundles endowed with a flat connection.

The contents of the article are as follows. In the next section we sketch a general method to obtain all principal fibre bundles admitting a flat connection over a given manifold. It is based on the following well known fact: The holonomy bundle, at any point of a flat principal fibre bundle over a manifold $M$ is a regular covering space of $M$. We also describe, as examples, the flat principal fibre bundle over a lens space (see Proposition 2.2) and over a surface of revolution embedded in $\mathbb{R}^{3}$ (see Propositions 2.4 and 2.5).

In Section 3 we study some properties of generalized Kaluza-Klein metrics on a principal fibre bundle $P(M, G)$, including, as a particular case, the Kaluza-Klein (also called bundle like) metrics. A generalized Kaluza-Klein metric on $P(M, G)$ is very rich in isometries. In fact the natural action of $G$ on $P$, which yields $M$ as orbit space, is carried out by isometries of any generalized Kaluza-Klein metric on $P(M, G)$.

In Section 4 we state the main theorem of the paper, which, following Palais [16], gives an example of reduction of variables for a variational problem. This theorem can be explained as follows: The construction of Willmore-Chen submanifolds in conformal classes associated with generalized Kaluza-Klein metrics on $P(M, G)$ which do not break the $G$-symmetry, is reduced, via the principle of symmetric criticality, to the problem of finding closed curves in $M$ which are critical points of a functional of the type

$$
\mathcal{F}^{r}(\gamma)=\int_{\gamma}\left(\kappa^{2}\right)^{\frac{r+1}{2}} d s
$$

where $\kappa$ denotes the curvature function of the closed curve $\gamma$ for a certain metric on $M$ and $r$ is the dimension of $G$. We will refer to critical points of this functional as $r$-generalized elasticae. When $r=1$, one has the classical notion of elastica, or free elastica (see [15]). We give interesting examples of generalized elasticae in Section 5. We first exhibit, for any natural number $m$, a rational one-parameter family of $m$-generalized closed elastic helices in any standard lens space (see Corollary 5.8). Next we discuss elasticity for parallels in a surface of revolution embedded in either the Euclidean 3-space $\mathbb{E}^{3}$ or Lorentz-Minkowskian 3 -space $\mathbb{L}^{3}$ (in this case we take timelike profile curves, so that parallels are always spacelike). In both cases we obtain the complete classification of surfaces of revolution, all of whose parallels are $m$-generalized elasticae. Besides right circular cylinders (all of whose parallels are geodesics and are hence $m$-generalized elasticae), there are Riemannian and 
Lorentzian trumpets of order $m$ (see Propositions 5.9 and 5.10, also Figures 5.1 and [5.2). The classical case $m=1$ was studied in [5].

Finally, in the last section, we obtain some applications relative to the existence of $G$-invariant Willmore-Chen submanifolds of Kaluza-Klein conformal structures (see Corollaries 6.11 to 6.17). We wish to point out the statements of Corollaries 6.15 and 6.17. For example, Corollary 6.15 shows that on any flat $G$-principal fibre bundle equipped with a Lorentzian Kaluza-Klein metric over the Lorentzian trumpets, there exists a codimension one foliation with constant mean curvature spacelike leaves, which are Willmore-Chen hypersurfaces.

\section{Flat Connections on a PRincipal fibre Bundle. Examples}

Let $M$ be a differentiable manifold and consider $P(M, G)$ a principal fibre bundle with base space $M$ and structure group $G$. Throughout this paper we will assume that $G$ is compact. Let $\Gamma$ denote a principal connection on $P$ with connection 1 -form $\omega$ which takes values in the Lie algebra $\mathfrak{g}$ of $G$. The connection $(\Gamma, \omega)$ is said to be flat if and only if its curvature form $\Omega$ vanishes identically. Then $(\Gamma, \omega)$ and the canonical flat connection locally agree, and this equality globally holds if the manifold is assumed to be simply connected. Said otherwise, if $M$ is simply connected and $(\Gamma, \omega)$ is flat, then $P=M \times G$ and $(\Gamma, \omega)$ coincides (up to isomorphisms) with the canonical flat connection on $M \times G$.

The class of principal fibre bundles which admit a flat connection can be described as follows. Let $\widetilde{M}$ be a nontrivial regular covering space of $M$ and let $p: \widetilde{M} \rightarrow M$ and $H$ be the covering map and the deck transformation group, respectively. It is well known [13, vol. I, p. 61] that $\tilde{M}(M, H)$ is a principal fibre bundle which admits a trivial flat connection, into $\left(\Gamma_{0}, \omega_{0}\right)$. We choose a monomorphism $\phi$ from $H$ in a Lie group $G$ and extend the transition functions $\left\{\psi_{k \ell}: U_{k} \cap U_{\ell} \rightarrow H\right\}$ of $\widetilde{M}(M, H)$, through $\phi$, to obtain $\left\{\bar{\psi}_{k \ell}=\phi \circ \psi_{k \ell}: U_{k} \cap U_{\ell} \rightarrow G\right\}$. These functions can be used as transition functions to define a principal fibre bundle $P(M, G)$ and $\phi$ can be extended to a monomorphism $\bar{\phi}$ from $\widetilde{M}$ to $P$ which maps $\left(\Gamma_{0}, \omega_{0}\right)$ in a flat connection on $P[13$, vol. I, p. 79].

The converse is also true. Indeed, given $P(M, G)$ and a flat connection $(\Gamma, \omega)$ on $P$, then the holonomy bundle $P_{u_{0}}$ through a point $u_{0} \in P$ is a regular covering space of $M$ [13, vol. I, p. 93], and it allows us to obtain $P(M, G)$ as above.

Next we give some examples for later use.

Example 2.1 (Principal fibre bundles with flat connections over a lens space). Let $\mathbb{S}^{2 n-1} \subset \mathbb{C}^{n}$ be the $(2 n-1)$-dimensional sphere of radius one, i.e.,

$$
\mathbb{S}^{2 n-1}=\left\{z=\left(z_{1}, \ldots, z_{n}\right) \in \mathbb{C}^{n}:|z|^{2}=\sum_{j=1}^{n}\left|z_{j}\right|^{2}=1\right\} .
$$

For any natural number $r$, let $\epsilon=e^{2 \pi i / r}$ be a primitive $r$-th root of unity and $\left\{s_{1}, \ldots, s_{n}\right\}$ integers which are relatively prime to $r$. We define an action of $\mathbb{Z}_{r}=$ $\left\{1, \epsilon, \epsilon^{2}, \ldots, \epsilon^{r-1}\right\}$ on $\mathbb{S}^{2 n-1}$ by

$$
\epsilon \cdot\left(z_{1}, \ldots, z_{n}\right)=\left(\epsilon^{s_{1}} z_{1}, \ldots, \epsilon^{s_{n}} z_{n}\right) .
$$

The orbit space is denoted by $L\left(r, s_{1}, \ldots, s_{n}\right)$ and it will be called a lens space. The natural projection $p: \mathbb{S}^{2 n-1} \rightarrow L\left(r, s_{1}, \ldots, s_{n}\right)$ gives the universal covering of this space. Hence $\mathbb{Z}_{r}$ is not only the fundamental group of $L\left(r, s_{1}, \ldots, s_{n}\right)$, but also the 
deck transformation group of this covering space. The classical case appears when $n=2$ and $L(r, 1, s)$ is usually denoted by $L(r, s)$. In particular, $L(2,1)$ is just the real projective space $\mathbb{R} P^{3}$.

Let $\left\{\psi_{k \ell}\right\}$ and $\omega_{0}$ be the transition functions of $\mathbb{S}^{3}\left(L(r, s), \mathbb{Z}_{r}\right)$ and the connection 1 -form of its canonical flat principal connection, respectively. For any compact Lie group $G$ endowed with a bi-invariant metric $d \sigma^{2}$, we choose an arbitrary closed geodesic through the identity of $G$, into $\beta(t)=\exp (t A)$, where $A \in \mathfrak{g}$. We define a monomorphism $\phi_{\beta}: \mathbb{Z}_{r} \rightarrow G$ by identifying $\mathbb{Z}_{r}$ with the group of primitive $r$-th roots of unity and then using that the exponential mapping defines an isomorphism between $\mathbb{S}^{1}$ and $\beta$. We may then extend $\left\{\psi_{k \ell}\right\}$ via $\phi_{\beta}$ to obtain a set of $G$-valued functions which can be used to construct a principal fibre bundle $P(L(r, s), G)$. Furthermore, $\phi_{\beta}$ is extended to get a monomorphism $\bar{\phi}_{\beta}: \mathbb{S}^{3} \rightarrow P$ which maps $\omega_{0}$ in a flat connection on $P$. Summing up, we have obtained the following result.

Proposition 2.2. Let $G$ be a compact Lie group. Then there exists a G-principal fibre bundle $P(L(r, s), G)$ over the lens space $L(r, s)$ which admits a principal flat connection with holonomy subbundle isomorphic to the 3-sphere, that is, $\mathbb{S}^{3}\left(L(r, s), \mathbb{Z}_{r}\right)$.

This proposition is also true if $G$ is not compact and the construction can also be generalized to lens spaces of higher dimensions.

Example 2.3. (Principal fibre bundles with flat connections over a surface of revolution). The fundamental group $\pi_{1}(M)$ of a surface of revolution $M$ embedded in $\mathbb{R}^{3}$ is free abelian with one or two generators according to whether the profile curve of $M$ is not closed or closed, respectively. We will discuss both cases separately.

2.1. Nonclosed profile curve $\left(\pi_{1}(M)=\mathbb{Z}\right)$. Let $\widetilde{M}$ be a regular covering space of $M$. Unless $\widetilde{M}$ is the universal covering of $M$, there exists an integer $k$ such that the deck transformation group $H$ of $p: \widetilde{M} \rightarrow M$ is $\mathbb{Z}_{k}$. Hence, given a compact Lie group $G$, a similar argument to that used in Example 2.1 can be used to obtain examples of $G$-principal fibre bundles over $M$ endowed with a principal flat connection whose holonomy is isomorphic to $\widetilde{M}$.

Let us assume that $\widetilde{M}$ is the universal covering of $M$, so it is diffeomorphic to the plane $\mathbb{R}^{2}$. The group $H=\mathbb{Z}$ certainly acts as a structure group of the principal fibre bundle $\widetilde{M}(M, \mathbb{Z})$. Let $\eta$ be a real number such that $\eta / \pi \notin \mathbb{Q}$ (the set of rational numbers), then the map $\phi_{\eta}: \mathbb{Z} \rightarrow \mathbb{S}^{1}$ given by $\phi_{\eta}(k)=e^{i k \eta}$ defines a monomorphism between $(\mathbb{Z},+)$ and $\mathbb{S}^{1} \subset \mathbb{C}$ regarded as a multiplicative group. Since every compact Lie group admits closed geodesics, we can extend $\phi_{\eta}$ to a monomorphism, also called $\phi_{\eta}$, from $\mathbb{Z}$ to $G$. Then, by applying the method established at the beginning of this section, we can obtain the following result.

Proposition 2.4. Let $M$ be a noncompact surface of revolution embedded in $\mathbb{R}^{3}$ and let $G$ be any compact Lie group. Then the following assertions hold:

(1) For any natural number $k$, there exists a $G$-principal fibre bundle $P_{k}(M, G)$ which admits a principal flat connection whose holonomy subbundle is isomorphic to $\widetilde{M}\left(M, \mathbb{Z}_{k}\right)$.

(2) For any real number $\eta$ such that $\eta / \pi \notin \mathbb{Q}$, there exists a $G$-principal fibre bundle $P_{\eta}(M, G)$ which admits a principal flat connection whose holonomy subbundle is isomorphic to $\mathbb{R}^{2}(M, \mathbb{Z})$. 
2.2. Closed profile curve $\left(\pi_{1}(M)=\mathbb{Z} \times \mathbb{Z}\right)$. Let $\widetilde{M}$ be a regular covering space of $M$, then the deck transformation group $H$ is (up to isomorphisms) either $\mathbb{Z}_{k} \otimes \mathbb{Z}_{\ell}$, or $\mathbb{Z} \otimes \mathbb{Z}_{\ell}$ or $\mathbb{Z} \otimes \mathbb{Z}$, where the last case appears when $\widetilde{M}$ is the universal covering.

Proposition 2.5. Let $M$ be a compact surface of revolution embedded in $\mathbb{R}^{3}$ and let $G$ be any compact Lie group with $\operatorname{dim} G>1$. For any pair of natural numbers $k$ and $\ell$ there exists a $G$-principal fibre bundle $P_{k \ell}(M, G)$ which admits a principal flat connection whose holonomy subbundle is isomorphic to either

(1) a torus if the holonomy subgroup is finite, that is, $H=\mathbb{Z}_{k} \otimes \mathbb{Z}_{\ell}$,

(2) a right circular cylinder if the holonomy subgroup is free abelian with one generator, that is, $H=\mathbb{Z} \otimes \mathbb{Z}_{\ell}(k=0)$, or

(3) a plane if the holonomy subgroup is free abelian with two generators, that is, $H=\mathbb{Z} \otimes \mathbb{Z}(k=\ell=0)$.

Proof. We write $H=H_{1} \otimes H_{2}$ to unify the three cases. Given any compact Lie group $G(\operatorname{dim} G>1)$ we are going to construct a monomorphism $\phi: H \rightarrow G$. To do this, let $\delta_{1} \equiv \delta_{1}(t)=\exp \left(t A_{1}\right), A_{1} \in \mathfrak{g}$, be a closed one-parameter subgroup in $G$. Then we map $H_{1}$ (via $\phi_{1}$ ) monomorphically in $\delta_{1}$ as we did in the last subsection. Now choose another closed one-parameter subgroup $\delta_{2} \equiv \delta_{2}(s)=\exp \left(s A_{2}\right), A_{2} \in \mathfrak{g}$, such that $A_{1}$ and $A_{2}$ are linearly independent. The existence of $\delta_{2}$ is guaranteed, because we can consider $\delta_{1}$ and $\delta_{2}$ as geodesics of a certain bi-invariant metric on $G$. As before, we map $H_{2}$ (via $\phi_{2}$ ) monomorphically in $\delta_{2}$. Finally, we move $\delta_{1}(t)$ through the image of $\phi_{2}$, that is, take $\phi_{2}\left(h_{2}\right) \cdot \delta_{1}(t)$ for all $h_{2} \in H_{2}$. Thus we obtain a family of integral curves associated with a left invariant vector field on $G$ which can be parametrized over $\mathrm{H}_{2}$. It is now clear that this allows us to define a monomorphism $\phi: H \rightarrow G$ and the proof is finished.

\section{The generalized KaluZa-Klein Structures AND THE Willmore-ChEN VARIATIONAL PROBlem}

Let $(\Gamma, \omega)$ be a principal connection on a principal fibre bundle $P(M, G), G$ being a compact Lie group endowed with a bi-invariant metric $d \sigma^{2}$. We denote by $\mathcal{M}$ and $\mathcal{F}_{+}$the spaces of pseudo-Riemannian metrics and smooth positive functions on $M$, respectively, and let $\overline{\mathcal{M}}$ denote the space of all pseudo-Riemannian metrics on $P$. For $\epsilon \in\{-1,+1\}$, let $\Phi_{\epsilon}: \mathcal{M} \times \mathcal{F}_{+} \rightarrow \overline{\mathcal{M}}$ be the mapping defined by

$$
\Phi_{\epsilon}(h, u)=\pi^{*}(h)+\epsilon(u \circ \pi)^{2} \omega^{*}\left(d \sigma^{2}\right),
$$

where $\pi: P \rightarrow M$ stands for the projection map of the principal fibre bundle.

A pseudo-Riemannian metric $\bar{h} \in \overline{\mathcal{M}}$ is called a generalized Kaluza-Klein metric on $P(M, G, \omega)$ (or on $P$, provided that there is no confusion) if it belongs to the image of the map defined above, that is, $\bar{h}=\Phi_{\epsilon}(h, u)$, for a certain metric $h$ and some positive function $u$.

By identifying each fibre of $P$ with the structure group $G$ (including metrics), a generalized Kaluza-Klein metric on $P$ can be viewed as a local warped product metric (see, for example, [11] and [18]). In particular, those generalized KaluzaKlein metrics obtained from constant functions $u$ in $\mathcal{F}_{+}$are called Kaluza-Klein metrics or "bundle like" metrics.

It is obvious that $\pi:(P, \bar{h}) \rightarrow(M, h)$ is a pseudo-Riemannian submersion whose leaves are the fibres, and so they are diffeomorphic to the structure group $G$. It is also evident that the natural action of $G$ on $P$ is carried out by isometries of $(P, \bar{h})$. 
For any pseudo-Riemannian metric $\tilde{h}$ on $P$, the Willmore-Chen variational problem can be stated as follows. Let $N$ be a compact smooth manifold of dimension $m+1$ and denote by $\mathcal{N}$ the space of immersions of $N$ in $P$. Then consider the submanifold $\mathcal{N}_{\tilde{h}}$ of $\mathcal{N}$ defined by

$$
\mathcal{N}_{\tilde{h}}=\left\{\phi \in \mathcal{N}: \phi^{*}(\tilde{h}) \text { is nondegenerate }\right\} .
$$

The Willmore-Chen functional $\mathcal{W}: \mathcal{N}_{\tilde{h}} \rightarrow \mathbb{R}$ is defined by

$$
\mathcal{W}(\phi)=\int_{N}\left(\alpha^{2}-\tau_{e}\right)^{\frac{m+1}{2}} d v
$$

$\alpha$ and $\tau_{e}$ being the mean curvature and the extrinsic scalar curvature functions of $\phi$, respectively, and $d v$ stands for the volume element of the induced metric $\phi^{*}(\tilde{h})$ on $N$. The critical points of $\mathcal{W}$ are called Willmore-Chen submanifolds and they only depend, as does the Willmore-Chen variational problem, on the conformal $\operatorname{class} \mathcal{C}(\tilde{h})$ of $\tilde{h}$.

\section{THE MAIN THEOREM}

In this section we deal with the Willmore-Chen variational problem associated with the conformal class $\mathcal{C}(\bar{h})$ of a generalized Kaluza-Klein metric $\bar{h}=\Phi_{\epsilon}(h, u)$ on $P(M, G, \omega)$. Our main theorem can be viewed as a variable reduction method for Willmore-Chen submanifolds. This argument gives a strong relation between this variational problem and a variational problem for closed curves in $\left(M, u^{-2} h\right)$.

For our purposes it is convenient to make the following conformal change in $(P, \bar{h})$. Define $\tilde{h}=(u \circ \pi)^{-2} \bar{h}$, so that

$$
\tilde{h}=\pi^{*}\left(u^{-2} h\right)+\omega^{*}\left(d \sigma^{2}\right) .
$$

It is easy to show that the projection map $\pi:(P, \tilde{h}) \rightarrow\left(M, u^{-2} h\right)$ has the following important properties:

(1) $\pi$ is a pseudo-Riemannian submersion and has totally geodesic fibres in $(P, \tilde{h})$.

(2) The natural action of $G$ on $P$ is carried out by isometries of $(P, \tilde{h})$.

(3) The submanifolds $\mathcal{N}_{\bar{h}}$ and $\mathcal{N}_{\tilde{h}}$ are the same, and the Willmore-Chen submanifolds of $(P, \bar{h})$ and $(P, \tilde{h})$ agree.

(4) For any $\phi \in \mathcal{N}\left(\mathcal{N}\right.$ standing for either $\mathcal{N}_{\bar{h}}$ or $\left.\mathcal{N}_{\tilde{h}}\right)$ and $a \in G$, we have $\mathcal{W}(\phi \cdot a)=\mathcal{W}(\phi)$, that is, $\mathcal{W}$ is $G$-invariant.

From now on we will denote by $\mathcal{S}$ the submanifold of $\mathcal{N}$ given by

$$
\mathcal{S}=\{\phi \in \mathcal{N}: \phi \cdot a=\phi \text { for all } a \in G\},
$$

that is, $\mathcal{S}$ consists of those immersions which are $G$-invariant. We also write down $\Sigma$ and $\Sigma_{G}$ to mean the set of critical points of $\mathcal{W}$ (i.e., the Willmore-Chen submanifolds) and $\left.\mathcal{W}\right|_{\mathcal{S}}$, respectively. Then we can apply the principle of symmetric criticality [16] to obtain $\Sigma \cap \mathcal{S}=\Sigma_{G}$. Therefore, in order to obtain Willmore-Chen submanifolds in $(P, \mathcal{C}(\bar{h}))$ which do not break the $G$-symmetry of the problem, we compute $\mathcal{S}$, then the restriction of $\mathcal{W}$ on $\mathcal{S}$ and then we proceed in due course.

Let $\gamma$ be a closed curve immersed in $M$. Then $N_{\gamma}=\pi^{-1}(\gamma)$ is an $(m+1)$ dimensional submanifold in $P$ which is $G$-invariant, $m$ being the dimension of $G$. The converse also holds. In fact, for any $(m+1)$-dimensional compact submanifold $N$ of $P$ which is $G$-invariant, there exists a closed curve $\gamma$ immersed in $M$ such that 
$N=N_{\gamma}$. Furthermore, the submanifold $N_{\gamma}$ is embedded if and only if $\gamma$ has no self-intersections in $M$. Therefore, we have the following identification:

$$
\mathcal{S}=\left\{N_{\gamma}=\pi^{-1}(\gamma): \gamma \text { is a closed curve immersed in } M\right\}
$$

We now compute the restriction of $\mathcal{W}$ to $\mathcal{S}$. Since $\pi:(P, \tilde{h}) \rightarrow\left(M, u^{-2} h\right)$ has totally geodesic fibres, it is, in particular, a harmonic submersion. Then the following relationship between the mean curvature function $\alpha$ of $N_{\gamma}$ in $(P, \tilde{h})$ and the curvature function $\kappa$ of $\gamma$ in $\left(M, u^{-2} h\right)$ holds (see [1])

$$
\alpha^{2}=\frac{1}{(m+1)^{2}}\left(\kappa^{2} \circ \pi\right) \text {. }
$$

As for the extrinsic scalar curvature $\tau_{e}$ of $N_{\gamma}$, we define a mapping $\Psi:[0, L] \times G \rightarrow$ $N_{\gamma}$ by

$$
\Psi(s, a)=\bar{\gamma}(s) \cdot a,
$$

$L>0$ being the length of $\gamma$ in $\left(M, u^{-2} h\right)$, where $\bar{\gamma}$ denotes a horizontal lift of $\gamma$ to $(P, \tilde{h})$. This certainly defines an isometry and allows us to define a global frame of unit vector fields on $N_{\gamma}$.

First, we choose $\left\{V_{1}, \ldots, V_{m}\right\}$ to be the fundamental vector fields in $P$ associated to a frame of unit left-invariant vector fields in $\left(G, d \sigma^{2}\right)$. They span the vertical distribution of $P$ and give an orthonormal frame on the fibre through every point of $P$. Now let $V_{0}$ be the horizontal lift of $\gamma^{\prime}(s)$. It is clear that $V_{0}$ is tangent to the horizontal lifts of $\gamma$. Hence $\left\{V_{0}, V_{1}, \ldots, V_{m}\right\}$ provides a global frame of unit vector fields on $N_{\gamma}$.

Let $K$ and $\tilde{K}$ be the sectional curvature functions of $N_{\gamma}$ and $(P, \tilde{h})$, respectively, restricted to the Grassmannian of 2-planes tangent to $N_{\gamma}$. Then we have

$$
\tau_{e}=\frac{1}{m(m+1)} \sum_{i, j=0}^{m}\left(K\left(V_{i}, V_{j}\right)-\tilde{K}\left(V_{i}, V_{j}\right)\right) .
$$

It is easy to see that $K\left(V_{0}, V_{j}\right)=0, j=1, \ldots, m$, because of the pseudo-Riemannian product structure of $N_{\gamma}$. Furthermore, by using the flatness of $(\Gamma, \omega)$, it is not difficult to show that $\tilde{K}$ vanishes over mixed (also called 'vertizontal', [19]) sections, that is, $\tilde{K}\left(V_{0}, V_{j}\right)=0, j=1, \ldots, m$ (see also [12]). Hence we have

$$
\tau_{e}=\frac{1}{m(m+1)} \sum_{i, j=1}^{m}\left(K\left(V_{i}, V_{j}\right)-\tilde{K}\left(V_{i}, V_{j}\right)\right) .
$$

Now it should be noticed that each fibre is totally geodesic not only in $(P, \tilde{h})$ but also in $N_{\gamma}$. Thus we may combine both Gauss equations to deduce that $\tau_{e}$ vanishes identically. Then we have

$$
\mathcal{W}\left(N_{\gamma}\right)=\int_{\gamma \times G}\left(\alpha^{2}\right)^{\frac{m+1}{2}} d s d A,
$$

$d A$ being the volume element of $\left(G, d \sigma^{2}\right)$, so that

$$
\mathcal{W}\left(N_{\gamma}\right)=\frac{\operatorname{vol}\left(G, d \sigma^{2}\right)}{(m+1)^{m+1}} \int_{\gamma}\left(\kappa^{2}\right)^{\frac{m+1}{2}} d s .
$$

The above computations suggests that we study the functional

$$
\mathcal{F}^{r}(\gamma)=\int_{\gamma}\left(\kappa^{2}\right)^{\frac{r+1}{2}} d s
$$


acting on closed curves in a Riemannian manifold. The variational problem associated with $\mathcal{F}^{r}$ has been considered in [4]. In particular, the Euler-Lagrange equations characterizing the critical points of $\mathcal{F}^{r}$, called $r$-generalized elasticae, were computed there. It should be noticed that 1-generalized elasticae are nothing but the classical free elastica curves (see, for instance, [15]). Summing up, we have shown the following result.

Theorem 4.6. Let $G$ be an $m$-dimensional compact Lie group endowed with a biinvariant metric. Let $(\Gamma, \omega)$ be a flat principal connection on a principal fibre bundle $P(M, G)$. Let $\bar{h}=\Phi_{\epsilon}(h, u)$ be a generalized Kaluza-Klein metric on $P(M, G, \omega)$ and $\mathcal{C}(\bar{h})$ its conformal class. Given an immersed closed curve $\gamma$ in $M$, then $N_{\gamma}$ is a Willmore-Chen submanifold in $(P, \mathcal{C}(\bar{h}))$ if and only if $\gamma$ is an m-generalized elastica in $\left(M, u^{-2} h\right)$.

\section{Some eXamples of Generalized elasticae}

The Euler-Lagrange equations associated with the functional $\mathcal{F}^{m}$ were computed in 4 . When the ambient space is a real space form, they imply that $r$-generalized elasticae must be contained in a totally geodesic submanifold with dimension less than or equal to 3. To obtain examples of $r$-generalized elasticae in the standard 3 -sphere, the authors used the nice geometry of closed helices. In particular, we obtained the following.

Proposition 5.7 ([4]). For any natural number $m$, there exists a one-parameter family $\left\{\gamma_{m, q}\right\}, q$ being a nonzero rational number, of closed helices in the standard 3-sphere which are $m$-generalized elasticae.

Let $L(r, s)$ be a lens space and denote by $p: \mathbb{S}^{3} \rightarrow L(r, s)$ the natural covering mapping. If $\mathbb{S}^{3}$ is endowed with its canonical metric, then we can define a metric on $L(r, s)$ such that $p$ becomes a Riemannian covering. In this sense we can talk about standard or canonical lens spaces. Now the above mentioned family of $m$ generalized elasticae in $\mathbb{S}^{3}$ can be projected onto $L(r, s)$ to obtain the following.

Corollary 5.8. For any natural number $m$, there exists a rational one-parameter family $\left\{\bar{\gamma}_{m, q}\right\}, q \in \mathbb{Q}-\{0\}$, of m-generalized closed elastic helices in any standard lens space.

Next we consider a surface of revolution and discuss when its parallels are generalized elasticae. Let us consider $\mathbb{R}^{3}$ endowed with the metric $g_{\epsilon}=d x^{2}+d y^{2}+\epsilon d z^{2}$, $\epsilon= \pm 1$, where $\{x, y, z\}$ stand for the usual rectangular coordinates. Notice that $\left(\mathbb{R}^{3}, g_{\epsilon}\right)$ is just the Euclidean 3 -space $\mathbb{E}^{3}$ or the Lorentzian 3 -space $\mathbb{L}^{3}$, according to whether $\epsilon=+1$ or $\epsilon=-1$ holds, respectively.

In the $x z$-plane we take an arclength parametrized curve $\beta(s)=(x(s), 0, z(s))$, $a<s<b$. Let us assume $x(s)>0$ and $\left\langle\beta^{\prime}(s) \beta^{\prime}(s)\right\rangle=\epsilon$. Let $S_{\beta}$ be the surface of revolution obtained by rotating the profile curve $\beta$ around the $z$-axis, which is Riemannian or Lorentzian according to whether $\epsilon=+1$ or $\epsilon=-1$ holds, respectively. Each point of $\beta$ describes a parallel $\gamma_{s}(\theta), \theta$ being the rotation angle, which is always spacelike. It is not difficult to see that the curvature function $\kappa_{s}$ of $\gamma_{s}$ in $S_{\beta}$ and the Gaussian curvature $K$ of $S_{\beta}$ along $\gamma_{s}$ satisfy

$$
\left(\kappa_{s}(\theta)\right)^{2}=\frac{\left(x^{\prime}(s)\right)^{2}}{x(s)^{2}} \quad \text { and } \quad K(s, \theta)=-\epsilon \frac{x^{\prime \prime}(s)}{x(s)},
$$

respectively. 


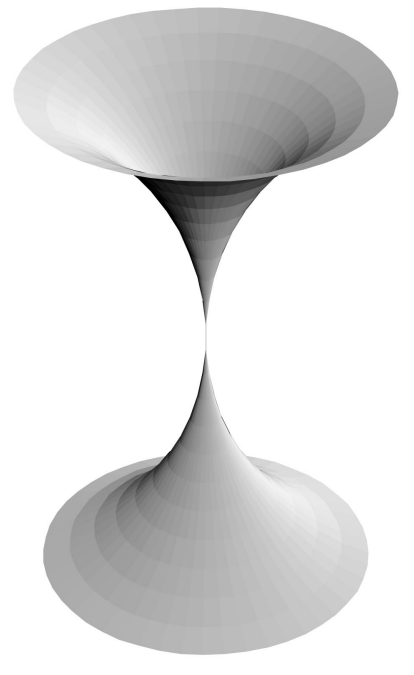

$R T_{1}$

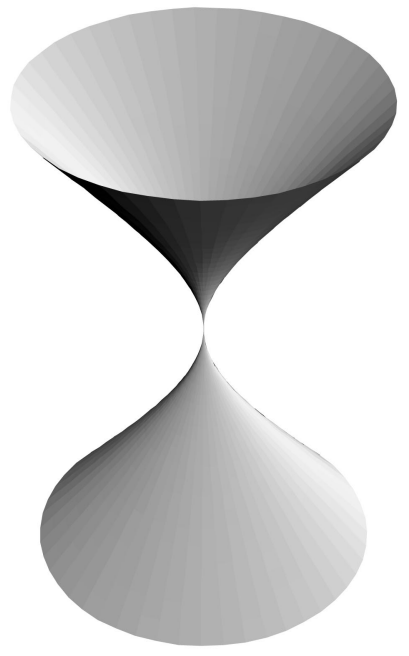

$L T_{1}$

Figure 5.1. Trumpets of order 1

On the other hand, we use the Euler-Lagrange equations associated with $\mathcal{F}^{m}$, [4], to deduce that a parallel $\gamma_{s}$ of $S_{\beta}$ is an $m$-generalized elastica if and only if

$$
\kappa_{s}^{2 m}\left(\epsilon m \kappa_{s}^{2}+(m+1) K\right)=0 .
$$

This equation implies that each geodesic parallel is always an $m$-generalized elastica, for any natural number $m$. This is also true for any closed geodesic. In this sense, these solutions will be called trivial critical points or trivial $m$-generalized elastic curves. Therefore, the nontrivial $m$-generalized elastic parallels are characterized by the following ordinary differential equation

$$
(m+1) x(s) x^{\prime \prime}(s)-m\left(x^{\prime}(s)\right)^{2}=0 .
$$

By using standard arguments, we can integrate that equation to obtain

$$
x(s)=A s^{m+1}, \quad A \text { being a certain constant, }
$$

where we have chosen the arclength parametrization to avoid the inclusion of unnecessary constants. Next we use $\left\langle\beta^{\prime}(s), \beta^{\prime}(s)=\epsilon\right\rangle$ to compute the $z$-coordinate of the profile curve,

$$
z(s)=\int_{0}^{s} \sqrt{1-\epsilon(m+1)^{2} A^{2} t^{2 m}} d t
$$

The surface of revolution whose profile curve is given by (5.1) and (5.2) will be called a Riemannian or a Lorentzian 'trumpet' of order $m$ according to whether $\epsilon=+1$ or $\epsilon=-1$ holds, respectively. We will denote them by $R T_{m}$ and $L T_{m}$, respectively (see Figures 5.1 and 5.2). Then we have

Proposition 5.9 (Riemannian case). A surface of revolution in the Euclidean 3space $\mathbb{E}^{3}$ has the property that all its parallels are m-generalized elastica if and only if it is either 

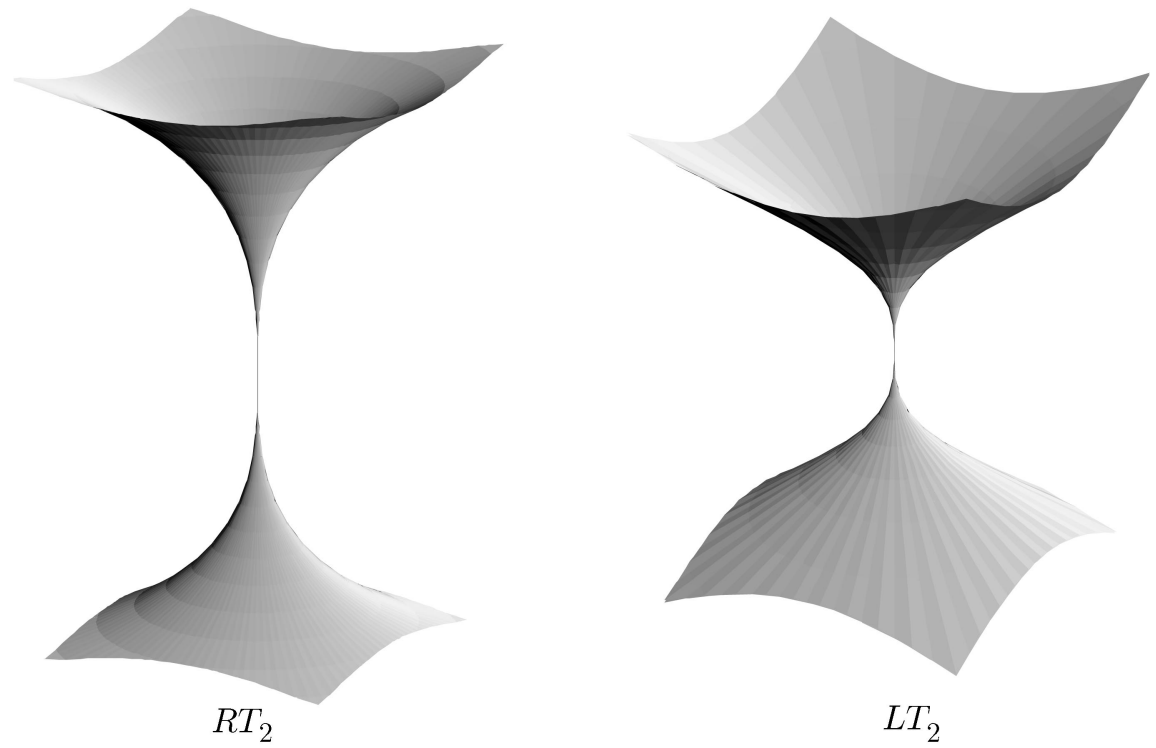

FiguRE 5.2. Trumpets of order 2

(1) a right circular cylinder, or

(2) a Riemannian trumpet of order $m$.

Proposition 5.10 (Lorentzian case). A surface of revolution in the Lorentzian 3space $\mathbb{L}^{3}$ has the property that all its parallels are m-generalized elastica if and only if it is either

(1) a right circular cylinder, or

(2) a Lorentzian trumpet of order $m$.

In view of the above results, we may study how the nontrivial $m$-generalized elastic parallels are distributed on the surface. A general analysis presents major difficulties, as one can see from [5], where a detailed study of this question has been made for $m=1$. For our purposes, it is enough to know that we can find surfaces of revolution in both $\mathbb{E}^{3}$ and $\mathbb{L}^{3}$ with qualitatively different distributions of their $m$-generalized elastic parallels. As an example, let us consider the circle of radius $r$ centered at the point $(a, 0,0) \in \mathbb{E}^{3}(a>r)$. We rotate it around the $z$-axis to obtain a torus of revolution. The region of hyperbolic points on this torus is given by $\beta(t)=\left(a-\sqrt{r^{2}-t^{2}}, t\right)$, where $t \in(-r, r)$. It is not difficult to see that there exist exactly two nongeodesic parallels which are $m$-generalized elastica, for any natural number $m$. Furthermore, these two parallels are symmetric with respect to the $x y$-plane.

\section{Some APplications}

In this section we will obtain nontrivial examples of Willmore-Chen submanifolds for Kaluza-Klein conformal structures on fibre bundles endowed with flat connections.

6.1. Flat principal fibre bundles on lens spaces. Let $g$ be the standard metric on the 3 -sphere $\mathbb{S}^{3}$ of radius 1 and project it to a lens space $L(r, s)$ to obtain its 
standard metric $h$. Thus $p:\left(\mathbb{S}^{3}, g\right) \rightarrow(L(r, s), h)$ is a Riemannian covering map. The following result can be proved by combining Proposition[2.2, Theorem 4.6] and Corollary 5.8 .

Corollary 6.11. Let $G$ be an $m$-dimensional compact Lie group. Then the following assertions hold:

(1) There exists a $G$-principal fibre bundle $P(L(r, s), G)$ over the lens space $L(r, s)$ which admits a principal flat connection $(\Gamma, \omega)$ with holonomy subbundle $\mathbb{S}^{3}\left(L(r, s), \mathbb{Z}_{r}\right)$.

(2) Let $d \sigma^{2}$ be a bi-invariant metric on $G$ and let $\bar{h}_{\epsilon}$ be the Kaluza-Klein metric on $P$ defined by

$$
\bar{h}_{\epsilon}=\pi^{*}(h)+\epsilon \omega^{*}\left(d \sigma^{2}\right),
$$

$\pi$ being the standard projection associated with $P$. Then for any natural number $m$ there exists a rational one-parameter family $\left\{N_{m q}\right\}, q \in \mathbb{Q}-\{0\}$, of $(m+1)$-dimensional submanifolds in $P$ satisfying the following properties:

(2.1) They are diffeomorphic to $\mathbb{S}^{1} \times G$.

(2.2) They have constant mean curvature in $\left(P, \bar{h}_{\epsilon}\right)$.

(2.3) They are Willmore-Chen submanifolds in $\left(P, \mathcal{C}\left(\bar{h}_{\epsilon}\right)\right)$.

Let us mention one interesting consequence of this corollary.

Corollary 6.12. Let $P$ be any $\mathbb{S}^{1}$-principal fibre bundle over $L(r, s)$ endowed with a flat connection $(\Gamma, \omega)$. Consider the metric $\bar{h}_{\epsilon}=\pi^{*}(h)+\epsilon \omega^{*}\left(d t^{2}\right)$. Then there exists a rational one-parameter family of Willmore tori in $(P, \mathcal{C}(\bar{h}))$ which has constant mean curvature in $\left(P, \bar{h}_{\epsilon}\right)$.

Remark 6.13. Our method of proof allows us to construct Willmore tori in the $\mathbb{S}^{1}$ principal fibre bundle $(P, \bar{h})$ defined above with non constant mean curvature. This construction works as follows. Let $\mathbb{S}^{2}$ be a unit 2-sphere totally geodesic in $\left(\mathbb{S}^{3}, g\right)$ and consider $S=p\left(\mathbb{S}^{2}\right)$, which is a totally geodesic surface in the lens space $L(r, s)$. In 15 Langer and Singer have shown that, up to rigid motions in $\mathbb{S}^{2}$, the family of closed free elasticae consists of a geodesic $\gamma_{0}$, into the equator, and a two-parameter family $\left\{\gamma_{a, b} \mid 0<a<b, a, b \in \mathbb{Z}\right\}$, where $\gamma_{a, b}$ means that the curve closes up after $a$ periods and $b$ trips around the equator $\gamma_{0}$. Therefore, the surface $S$ has plenty of free elasticae, which can be obtained by projecting via $p$ the above family of free elasticae in $\mathbb{S}^{2}$. Then $N_{a, b}=\pi^{-1}\left(p\left(\gamma_{a, b}\right)\right)$ is a Willmore torus with nonconstant mean curvature in $(P, \bar{h})$.

6.2. Flat principal fibre bundles over surfaces of revolution. Given an $m$ dimensional compact Lie group $G$, Proposition 2.4 guarantees the existence of two families of $G$-principal fibre bundles over a surface of revolution $S$ admitting a flat connection. When $S$ is chosen to be a trumpet (Riemannian or Lorentzian) of order $m$, endowed with its standard metric, then the associated Kaluza-Klein structure has nice properties which are detailed in the next statements.

Corollary 6.14. Let $G$ be any $m$-dimensional compact Lie group endowed with a bi-invariant metric $d \sigma^{2}$ and consider $P\left(R T_{m}, G\right)$ a $G$-principal fibre bundle which admits a principal flat connection $(\Gamma, \omega)$. Let $\bar{h}$ be the Kaluza-Klein metric given by $\bar{h}=\pi^{*}(h)+\omega^{*}\left(d \sigma^{2}\right), h$ and $\pi$ standing for the standard metric on $R T_{m}$ and the projection associated with $P$, respectively. Then there exists a codimension one 
foliation of $P$ whose leaves have constant mean curvature in $(P, \bar{h})$. Furthermore, they are Willmore-Chen hypersurfaces in $(P, \mathcal{C}(\bar{h}))$.

Proof. Pick a point $x$ in $P$ and consider the parallel $\gamma_{x}$ in $R T_{m}$ through $\pi(x)$. It follows easily that $\left\{N_{x}=\pi^{-1}\left(\gamma_{x}\right)\right\}_{x \in P}$ defines a codimension one foliation on $P$. Since $\pi:(P, \bar{h}) \rightarrow\left(R T_{m}, h\right)$ is a Riemannian submersion with totally geodesic fibres, (4.1) implies that each leaf $N_{x}$ has constant mean curvature in $(P, \bar{h})$. Furthermore, Theorem 4.6 and Proposition 5.9 imply that the leaves are WillmoreChen hypersurfaces in $(P, \mathcal{C}(\bar{h}))$.

To understand the Lorentzian case better, we write down $h_{L}$ to denote the standard Lorentzian metric on $L T_{m}$. Let $\bar{h}_{L}$ be the associated Kaluza-Klein metric on $P$, that is, $\bar{h}_{L}=\pi^{*}\left(h_{L}\right)+\omega^{*}\left(d \sigma^{2}\right)$, which is a Lorentzian metric.

Corollary 6.15. Let $P\left(L T_{m}, G\right)$ be a G-principal fibre bundle over a Lorentzian trumpet of order $m$ which admits a principal flat connection. Then there exists a codimension one foliation of $P$ whose leaves are constant mean curvature spacelike hypersurfaces in $\left(P, \bar{h}_{L}\right)$. Moreover, these leaves are Willmore-Chen hypersurfaces in $\left(P, \mathcal{C}\left(\bar{h}_{L}\right)\right)$.

Remark 6.16. The same method still works when we consider right circular cylinders (Riemannian or Lorentzian) instead of trumpets. In both cases parallels are spacelike geodesics and so $(P, \bar{h})$ admits a codimension one foliation whose leaves are spacelike and minimal. Furthermore, they are nontrivial Willmore-Chen hypersurfaces, because minimality implies the Willmore-Chen condition only when the ambient space is of constant curvature.

To finish this section we are going to give an application which can be considered representative of the kind of consequences that we can obtain in the compact case. Bearing in mind Proposition 2.5] let us assume $S$ is a torus, so that it contains a pair of $m$-generalized elastic parallels for any natural number $m$. Then the following result is clear.

Corollary 6.17. There exists a pair of Willmore-Chen hypersurfaces of constant mean curvature in the Kaluza-Klein conformal structure on any principal fibre bundle, endowed with a flat connection, over a torus of revolution.

\section{REFERENCES}

[1] M. Barros, Willmore tori in non-standard 3-spheres, Math. Proc. Cambridge Phil. Soc., 121, 321-324, 1997. MR 98b:53052

[2] M. Barros, Free elasticae and Willmore tori in warped product spaces, Glasgow Math. J., 40, 265-272, 1998. CMP 98:14

[3] M. Barros and B. Chen, Stationary 2-type surfaces in a hypersphere, J. Math. Soc. Japan, 39, 627-648, 1987. MR 89b:53091

[4] M. Barros, A. Ferrández, P. Lucas, and M. A. Meroño, Willmore tori and Willmore-Chen submanifolds in pseudo-Riemannian spaces, J. Geom. Phys., 28, 45-66, 1998. CMP 99:03

[5] M. Barros and O. J. Garay, Free elastic parallels in a surface of revolution, Amer. Math. Monthly, 103, 149-156, 1996. MR 96k:53002

[6] M. Barros and O. Garay, Hopf submanifolds in $\mathbb{S}^{7}$ which are Willmore-Chen submanifolds, Math. Z., 228, 121-129, 1997. MR 99d:53065

[7] M. Barros, O. Garay, and D. Singer, New examples of Willmore surfaces, Preprint, 1995.

[8] M. Barros and M. A. Meroño, Willmore tori in Kaluza-Klein conformal structures on the three sphere, Preprint, 1997.

[9] B. Chen. Some conformal invariants of submanifolds and their applications, Boll. Un. Mat. Ital., 10, 380-385, 1974. MR 51:6663 
[10] N. Ejiri, A counterexample for Weiner's open question, Indiana Univ. Math. J., 31, 209-211, 1982. MR 83j:53057

[11] A. Gray, Pseudo-Riemannian almost product manifolds and submersions, J. Math. Mech., 16, 715-737, 1967. MR 34:5018

[12] G. Jensen, Einstein metrics on principal fibre bundles, J. Diff. Geom., 8, 599-614, 1973. MR 50:5694

[13] S. Kobayashi and K. Nomizu, Foundations of Differential Geometry, John Wiley, New York, 1963 (I), 1969 (II). MR 27:2945 MR 38:501

[14] J. Langer and D. Singer, Curves in the hyperbolic plane and mean curvature of tori in 3-space, Bull. London Math. Soc., 18, 531-534, 1984. MR 85k:53006

[15] J. Langer and D. Singer, The total squared curvature of closed curves, J. Differential Geometry, 20, 1-22, 1984. MR 86i:58030

[16] R. Palais, The principle of symmetric criticality, Commun. Math. Phys., 69, 19-30, 1979. MR 81c:58026

[17] U. Pinkall, Hopf tori in $\mathbb{S}^{3}$, Inventiones mathematicae, 81, 379-386, 1985. MR 86k:53075

[18] A. Romero and M. Sánchez, New properties and examples of incomplete Lorentzian tori, J. Math. Phys., 35, 1992-1997, 1994. MR 95h:53093

[19] A. Weinstein, Fat bundles and symplectic manifolds Adv. Math., 37, 239-250, 1980. MR 82a:53038

Departamento de Geometría y Topología, Universidad de Granada, 18071 Granada, SPAIN

E-mail address: mbarros@ugr.es

Departamento de Matemáticas, Universidad de Murcia, 30100 Espinardo, Murcia, SPAIN

E-mail address: aferr@um.es

Departamento de Matemáticas, Universidad de Murcia, 30100 Espinardo, Murcia, SPAIN

E-mail address: plucas@um.es

Departamento de Matemáticas, Universidad de Murcia, 30100 Espinardo, Murcia, SPAIN

E-mail address: mamb@um.es 